\title{
Le financement de la formation post-secondaire*
}

\author{
AVIGDOR FARINE**, PIERRE-PAUL PROULX† ET CLÉMENT LEMELIN††
}

\section{RÉSUMÉ}

Ce travail présente certaines notions sur la formation post-secondaire et son financement. Les propositions portent sur les coûts monétaires, les coûts directs et indirects lle manque à gagner inclus) et sur l'aide financière aux étudiants. L'article passe en revue les bénéficiaires de la formation post-secondaire et leur contribution au financement de cette formation. Il est évident que la société défraie en grande partie les coûts directs par l'intermédiaire des gouvernements, et les étudiants les coûts indirects.

L'intervention de l'Etat est surtout liée à l'investissement en éducation, à la création des biens culturels et à l'aide financière aux étudiants pour suppléer les imperfections du marché du capital. D'ailleurs, les programmes de bourses devraient orienter les étudiants vers les domaines d'études prioritaires du point de vue social et économique.

Tenant compte du manque à gagner, l'apport des étudiants au financement de leur formation est très important. Cependant, étant les principaux bénéficiaires de cette formation (la rentabilité privée de la formation post-secondaire demeure en général évidente), il est raisonnable d'admettre que ceux qui bénéficient de cette formation sous forme de revenus élevés, devraient supporter un pourcentage plus élevé des coûts institutionnels. Un moyen de le faire serait un régime de prêts plus élevés et remboursables sur une période prolongée.

\section{ABSTRACT \\ Financing Post-Secondary Education}

Higher education occupies a dominant place in the public sector. This paper deals with the direct and indirect costs of post-secondary training, forgone earnings and direct expenditures

*Cet article s'appuie sur un rapport du Centre de recherche en développement économique (C.R.D.E.) préparé à l'intention du ministère de l'Education au début de 1975. France Laurendeau, Noël Bouliane et T.P. Loc, assistants de recherche au C.R.D.E. avaient participé à la préparation du rapport.

**Professeur à la Faculté des sciences de l'éducation, Université de Montréal.

$\nmid$ Professeur au Département de sciences économiques, Université de Montréal.

$\dagger †$ Professeur au Département de sciences économiques, UQUAM. 
of students, and student aid programs. It is evident that society at large covers most of the direct costs through the different Government levels, and students the indirect ones (forgone earnings).

Government intervention is related to education as investment, the allocation of resources, methods of financing, and student aid programs.

Considering the forgone earnings, the resource contribution by students is very important. However, being the main beneficiaries of postsecondary training, it is reasonable to say that those who benefit by that training by receiving higher incomes, should bear a higher percentage of the institutional costs. To that purpose, a contingent repayment student assistance program seems appropriate.

In other respects, in order to interest students in fields of study having social and economic priorities, more scholarships should be made available in them.

\section{Introduction}

Au cours de la dernière décennie, l'enseignement post-secondaire au Canada, en général, et au Québec, en particulier, a connu une expansion considérable. L'accroissement de la population étudiante au Québec est due, en partie, à la création des Cegeps qui a favorisé l'accès aux études universitaires plus que ne le faisaient les anciens collèges classiques menant au Baccalauréat. Pour montrer l'importance accordée à ce niveau d'enseignement, il suffit de mentionner que les subventions du ministère de l'Education aux universités ont augmenté à un taux annuel de 23\% durant la période 1961-1974, alors que celles reçues par les Cegeps ont atteint $24.3 \%$ par an entre 1968 et $1974 .^{1}$

Depuis quelques années, on s'interroge à l'égard du financement de la formation postsecondaire. ${ }^{2}$ Nous en voulons pour preuve: la prise de position des étudiants du Québec en 1972-73, notamment ceux de l'UQUAM, contre les frais de scolarité, et la grève dans les Cegeps en automne 1974. Ces événements peuvent bien se situer dans la perspective d'une lutte pour l'accessibilité aux études post-secondaires, susceptible de conduire en conséquence à la clarification du rôle social de l'université. N'a-t-on pas enregistré de semblables protestations en Ontario et au Nouveau-Brunswick en 1975-76?

Cet article présente certaines notions de base concernant la formation post-secondaire et discute de la participation des divers agents économiques (l'étudiant, la famille, l'entreprise privée et l'Etat) au financement de cette formation, et des bénéfices qu'ils retirent. Ce travail essaie d'apporter des suggestions d'amélioration au système existant d'aide financière aux étudiants.

Notre démarche méthodologique consiste à:

- placer d'abord l'enseignement post-secondaire dans une perspective plus large de formation,

- préciser ensuite la nature des coûts auxquels les divers agents économiques font face,

- proposer une réponse à la question complexe des bénéfices des divers agents économiques,

- définir, enfin, les notions se rapportant à l'aide financière aux étudiants. 


\section{La nature de la formation post-secondaire}

Une personne peut fréquenter le Cegep ou l'université soit pour sa satisfaction immédiate, soit dans le but de se livrer plus tard à d'autres activités pour lesquelles le séjour dans ces institutions est utile. La première motivation en est une de consommation, et la deuxième d'investissement; cependant, la plupart du temps, les deux jouent simultanément. Retenons que toute activité s'accompagnant de bénéfices qui surviendront après les coûts est une activité d'investissement. En se référant à cette définition, on peut affirmer que l'enseignement post-secondaire est une activité d'investissement qui produit des effets durables: on s'attend à ce qu'il y ait un rapport positif entre le niveau d'instruction d'un individu et son revenu. De cette corrélation scolarité-revenu, on peut inférer des taux de rendement internes pour des années supplémentaires d'étude. Dans la plupart des études empiriques faites jusqu'ici, ce taux de rendement s'est avéré positif, même si le taux de rendement décroît avec le niveau de scolarité. Cependant, certaines études récentes montrent que le taux de rendement monétaire de certains types d'études universitaires peut être négatif. Ajoutons tout de suite que de façon générale, les termes revenu et taux de rendement devraient être utilisés dans un sens large et englober des aspects qualitatifs non-pécuniaires, ce que ne font pas ces études. De plus, une bonne interprétation des taux de rendement devrait s'assurer que le profil des revenus utilisés ne reflète pas des situations de déséquilibre, surtout en ce qui concerne les revenus en début de carrière. Bien que la plupart des études effectuées et les concepts examinés dans ce texte portent sur les divers niveaux d'enseignement formel, on peut aussi les appliquer à la formation sur le tas. Historiquement, on a pu voir l'entreprise privée céder progressivement certains domaines de formation à l'enseignement public, comme en témoignent les champs de spécialisation aux Cegeps. En plus, la formation formelle est de type général, pouvant servir à l'exercice d'une pluralité d'emplois, tandis que celle offerte sur le tas est en bonne partie spécifique, servant avant tout l'employeur qui défraie la presque totalité des coûts. ${ }^{3}$ Ces faits justifient en quelque sorte le sentiment de frustration qui hante les bénéficiaires d'une formation générale sur le tas lorsqu'ils se comparent à ceux qui fréquentent des institutions d'enseignement lesquels sont aidés largement par l'Etat. On sait que la formation en industrie privée à l'aide de fonds publics est peu développée au Québec.

Cette distinction entre divers types de formation nous permet de souligner certaines caractéristiques de la population étudiante. En effet, le cheminement des jeunes dans le système scolaire n'est plus linéaire. Un nombre croissant d'étudiants abandonnent temporairement leurs cours, changent de discipline ou bien s'inscrivent à temps partiel dans des Cegeps ou des universités. ${ }^{4}$ Le type de société dans lequel nous vivons actuellement postule la formation permanente.

\section{La definition des coûts}

La notion des coûts est très importante et afin de pouvoir soutenir notre argumentation, il s'avère nécessaire d'élaborer ici certains concepts. On accepte que dans la vie on ne peut tout avoir, qu'il faut choisir et choisir c'est sacrifier. Dans la mesure où l'on évalue ces sacrifices, on dérive des coûts. Il convient de s'aviser des moyens pour atteindre les objectifs au moindre coût ou d'adopter des projets dont les bénéfices dépassent avantageusement 
les coûts. De la comparaison des bénéfices et des coûts résulte le taux de rendement d'une activité. Ajoutons également que les transferts monétaires dans le financement de l'enseignement post-secondaire ne nous permettent pas toujours de définir avec précision les coûts, la monnaie n'étant qu'un étalon qui permet de tout ramener à une même unité. Dans le but de mieux préciser ces coûts, nous formulons trois propositions.

\section{Le coût total d'une année d'études n'est pas égal au cô̂t monétaire observé}

Le processus d'enseignement exige en effet l'utilisation de diverses ressources, parmi lesquelles se range le temps des étudiants consacré aux études. Ce dernier est une composante des coûts fort importante, même s'il ne s'accompagne pas d'un transfert monétaire. Pendant qu'il étudie, un jeune ne peut pas se livrer à d'autres activités. Mesuré par le manque à gagner, ce sacrifice est de taille. En effet, certaines études estiment que ce coût indirect peut correspondre entre 47 et $53 \%$ du coût total de l'enseignement post-secondaire, le reste étant le coût des facteurs traditionnels de production. ${ }^{5}$ C'est cette portion du coût (salaires, intérêt, frais de fonctionnement) que nous désignons par coût monétaire. Cependant, il n'est pas assuré que tous les étudiants pourraient concrétiser leur manque à gagner, s'ils se retrouvaient tous ensemble sur le marché du travail. ${ }^{6}$ D'autre part, certains ne sont pas loin d'affirmer que c'est ce manque à gagner et non les frais de scolarité qui constitue la barrière monétaire la plus discriminatoire à l'égard des jeunes issus de milieux défavorisés. ${ }^{7}$

2. Le coût monétaire de l'enseignement n'est pas égal aux frais encourus par les institutions d'enseignement

Il paraît intéressant d'établir une équation entre le coût monétaire de l'enseignement et la somme des frais de fonctionnement et des dépenses de capital des institutions d'enseignement. Il ne faudrait pas sous-estimer les autres frais tels que l'achat de volumes, les dépenses de transport, etc. auxquels font face les étudiants. Par ailleurs, les institutions d'enseignement post-secondaire sont des entreprises à produit multiple, et l'enseignement ne constitue qu'une des fonctions de ces institutions. Il suffit de penser à la recherche et à la mission, visée ou réelle, de rayonnement dans la société pour s'en convaincre. Si, par souci d'une plus grande rationalité on cherche à isoler et à mieux définir le coût de l'enseignement, il faut alors séparer les composantes du coût, tâche difficile à exécuter. ${ }^{8}$

\section{Le coût monétaire de l'enseignement n'est pas égal aux frais de scolarité}

Cette proposition est bien évidente et il s'agit ici de retenir que l'Etat aide l'étudiant non seulement par ses politiques d'aide financière à l'étudiant même, mais aussi par ses subventions aux institutions d'enseignement. Il faut souligner que dans la mesure où les frais de scolarité sont égaux dans les diverses facultés ou pour les divers niveaux d'études, l'assistance de l'Etat augmentera selon le coût monétaire plus élevé de certains programmes.

\section{L'aide financiere aux etudiants}

Un des thèmes de notre travail est qu'il faut situer l'examen des politiques de financement dans un contexte plus global d'intervention gouvernementale dans la formation post-secondaire. Il y a aide à l'étudiant dans la mesure oû les coûts auxquels il fait face ne sont pas égaux à ceux que sa formation entraîne. Nous partons de l'idée que l'étudiant devrait dé- 
frayer les coûts de sa formation dans la mesure où il en retire des bénéfices. Mais comment préciser et isoler certains des bénéfices de l'enseignement qui reviennent non à l'étudiant mais au reste de la société? En outre, les frais de scolarité ne correspondent pas au coût direct de la formation et la politique des subventions aux universités est une forme d'assistance aux étudiants fort importante. Si les étudiants défrayaient eux-mêmes les coûts directs de l'enseignement, les frais de scolarité différeraient beaucoup d'une faculté à une autre et d'un niveau à l'autre. Enfin, toute politique de gratuité scolaire n'abolirait qu'une partie des frais encourus par l'étudiant. Il reste toujours le manque à gagner dont on connaît l'importance.

L'aide aux étudiants se réfère, souvent inconsciemment, à ce manque à gagner. La bourse est un moyen par lequel l'Etat décide de défrayer une partie des coûts directs ou indirects (manque à gagner) de l'enseignement. Dans la mesure où cette bourse est supérieure aux frais de scolarité, on peut supposer que l'Etat reconnaît l'existence des coûts indirects. Quant au prêt, il s'agit là d'une assistance limitée. Il existe évidemment un transfert dans la mesure où l'intérêt n'est pas payé par l'étudiant ou que l'intérêt payé diffère de beaucoup de celui qui aurait été payé par l'étudiant sans l'intervention gouvernementale.

Avant de passer au financement de la formation post-secondaire, nous pouvons dire que les motifs de l'intervention de l'Etat, et les objectifs visés par ses modes de financement sont liés à l'aspect investissement en éducation et à la création de biens culturels. L'éducation contribue au développement des ressources humaines et à la création d'une maind'oeuvre hautement qualifiée requise pour le développement économique et social. L'Etat a également pour but d'encourager l'accessibilité à la formation post-secondaire et de promouvoir la mobilité sociale qui en résulte.

\section{Le financement de la formation post-secondaire, beneficiaires et payeurs}

Pour mieux comprendre la participation des divers agents économiques au financement de cette formation, il est bon de définir les bénéfices qu'ils en retirent avant de décrire les diverses formes de leur participation aux couts.

\section{Les bénéficiaires de la formation post-secondaire}

a) L'enseignement représente la fonction majeure du Cegep et de l'université. Selon une enquête récente menée aux Etats-Unis, $75 \%$ des professeurs s'intéressent principalement à l'enseignement. ${ }^{9}$ Dans une optique de consommation et d'investissement, l'enseignement rapporte des profits essentiellement à l'étudiant. La satisfaction retirée du savoir acquis au présent ou des revenus élevés à l'avenir, un statut social plus enviable et un style de vie plus conforme à leurs aspirations, représentent ces bénéfices de l'enseignement.

b) Dans une optique d'investissement, l'enseignement a aussi des effets sur le reste de la société. Il forme une main-d'oeuvre hautement qualifiée dont les services sont utilisés, contre rémunération, par le reste de la société.

c) La recherche scientifique aide directement l'étudiant en lui permettant d'approfondir la discipline dans laquelle il désire se spécialiser.

d) Comme la recherche contribue au progrès technique, elle a une portée sociale. Elle 
représente un facteur du développement économique et de la croissance dont toute la société bénéficie.

e) Le rayonnement des institutions post-secondaires est bénéfique essentiellement à la société. Il s'agit de biens culturels dont les étudiants bénéficient, généralement au même titre que les autres membres de la société.

\section{Les sources de financement}

a) Si l'on veut bien pousser la logique des marchés jusqu'à la limite, on peut dire que les institutions post-secondaires produisent des diplômés qu'elles ne peuvent cependant pas, contrairement à la plupart des autres firmes, "vendre" pour financer leurs opérations. Cependant, on pourrait également dire qu'elles produisent de l'enseignement qu'elles pourraient "vendre" aux étudiants.

b) Les étudiants paient des frais de scolarité aux institutions (sauf au Cegep). En 19731974, ces frais s'élevaient à $14 \%$ des coûts institutionnels. ${ }^{10}$ De plus, les étudiants paient indirectement par le manque à gagner, et défraient certaines dépenses spécifiques (volumes, transport).

c) Un grand nombre d'étudiants reçoivent de l'aide de leurs parents sous forme de nourriture et logement gratuits. En plus, les parents aident leurs fils et filles à payer leurs frais de scolarité et autres dépenses (vêtements, transport, loisirs). Dans le cas d'un étudiant marié, son conjoint (s'il n'est pas lui-même étudiant), joue parfois un rôle similaire à celui des parents dans l'aide qu'il accorde.

d) Les entreprises privées, les organismes religieux et les particuliers qui font des dons aux institutions, contribuent au financement de la formation post-secondaire, contribution qui s'annonce meilleure et dont une partie est publique à cause des déductions d'impôt permises.

e) Les étudiants peuvent bénéficier des fonds de recherche provenant du gouvernement fédéral, en tant que techniciens et assistants de recherche. Les prêts aux étudiants ont leur source dans les programmes d'aide du fédéral. En outre, les exemptions fiscales permises dans le calcul de l'impôt sur le revenu, la taxe de vente réduite pour le matériel scolaire et des projets tels que Perspective Jeunesse et Initiatives Locales représentent d'autres formes d'aide à l'étudiant.

f) Aussi surprenant que cela puisse paraître, le gouvernement fédéral défraie finalement plus de la moitié des dépenses institutionnelles encourues.

g) Le gouvernement provincial demeure l'organisme public le plus directement engagé dans la promotion de la formation post-secondaire. Il subventionne les Cegeps et les universités en participant à leurs frais d'exploitation et d'immobilisation. En outre, divers ministères accordent des subventions de recherche. Par le système de Prêts et Bourses et par son programme de Placement Etudiant, le gouvernement permet à un grand nombre d'étudiants de poursuivre leurs études.

En résumé, c'est la société qui défraie en grande partie les coûts directs par l'intermédiaire des gouvernements, tandis que les étudiants se chargent plutôt des coûts indirects.

\section{Quelques remarques sur le financement de la formation post-secondaire}

Dans les paragraphes qui suivent, nous faisons quelques remarques sur l'état actuel du financement de cette formation. 


\section{Les préoccupations de l'Etat}

a) La montée des coûts est une source de préoccupation pour l'Etat. L'éducation, la santé, les arts, sont des secteurs où le progrès technologique semble être relativement difficile à atteindre, ce qui cause l'appropriation d'une part grandissante du revenu national.

b) Un des objectifs des politiques fiscales et monétaires est de transférer des avoirs des riches vers les pauvres. Il n'est pas assuré que les politiques d'aide aux étudiants atteignent cet objectif. Les résultats d'études sur l'accessibilité à l'enseignement post-secondaire ici, ou ailleurs, rendent le lecteur moins optimiste quant aux effets redistributifs des politiques de financement. ${ }^{11}$ On est porté à s'interroger quand on sair que des fonds prélevés sur l'ensemble de la population sont distribués à des jeunes qui occuperont demain des postes bien rémunérés et qui, même aujourd'hui, sont souvent issus de milieux favorisés. Il faut noter également qu'une bonne partie de l'aide à la formation, la partie qui passe par les subventions aux institutions post-secondaires, n'est pas discriminatoire: le revenu des parents ne détermine en rien les montants alloués. Néanmoins, les politiques d'aide aux étudiants permettent à plusieurs jeunes venant de milieux moins favorisés de poursuivre leur formation et d'atteindre ainsi des objectifs de mobilité sociale.

c) Nous avons vu précédemment que l'existence de biens culturels encourage l'intervention de l'Etat: certains bénéfices sont appropriés par l'ensemble de la société. C'est là une raison d'efficacité, mais il ne faudrait pas en rester là. En effet, pour la collectivité, certains types de formation semblent plus rentables que d'autres et l'Etat devrait davantage favoriser ces secteurs. Ceci nécessiterait, cependant, une planification sur une période plus prolongée.

\section{Remarques à l'égard de l'étudiant}

a) La notion de dépendance des parents semble créer un "problème" pour les étudiants qui souhaiteraient être traités autrement. Or, les critères d'attribution des prêts et bourses suppoșent explicitement que les parents défrayent une partie des dépenses de leurs enfants. Il est difficile d'imaginer des politiques d'aide aux étudiants visant à aider les jeunes issus de milieux moins favorisés, et ne tenant pas compte du statut socio-économique des parents. Il est cependant possible de traiter les bourses différemment des prêts. Si les prêts et bourses possédaient un seuil visant à assurer la subsistance de tous les étudaints, toute somme supplémentaire versée aux étudiants venant des milieux moins favorisés n'aurait pas pour but de subvenir aux besoins élémentaires de ces jeunes mais jouerait plutôt un rôle d'incitation à la poursuite des études. Nous concluons, cependant, que les facteurs qui déterminent la poursuite des études (facteurs personnels, familiaux et culturels) sont tels qu'il ne faut pas accorder aux prêts et aux bourses un rôle trop important dans la prise de décision concernant les études.

b) Les critères d'attribution des prêts et bourses supposent explicitement que l'étudiant travaille l'été. Il s'agit d'une bonne chose en soi, dans la mesure où l'étudiant y trouve l'occasion d'appréhender le monde du travail d'une façon plus réaliste. Mais le nombre d'emplois d'été semble insuffisant.

c) L'argumentation habituelle en faveur d'une politique d'aide à partir des prêts met l'accent sur la taille des revenus des futurs diplômés. L'Etat intervient pour éliminer les difficultés causées par le mauvais fonctionnement du marché du capital. Une fois sur 
le marché du travail, il ne devrait pas être difficile de remettre intérêt et pricipal, dans la mesure où la période de remboursement est prolongée. C'est d'ailleurs sur le rythme de remboursement que portent plusieurs des critiques du système actuel.

d) Beaucoup d'étudiants pensent que les frais de scolarité créent une barrière à l'accessibilité de la formation post-secondaire. C'était le principal objet de la grève des étudiants en 1972-1973. Il ne nous semble pas que les frais de scolarité constituent la barrière la plus importante. Les résultats scolaires causent bien plus d'abandons des études. ${ }^{12} \mathrm{De}$ plus, ces frais de scolarité qui sont demeurés constants depuis quelques années, se déprécient en termes réels du fait de l'inflation et constituent une part décroissante des revenus des institutions.

\section{Vers un regime de prets remboursables?}

L'idée d'un régime de prêts remboursables en proportion du revenu n'est pas nouvelle. Récemment, divers projets ont vu le jour aux Etats-Unis tels que le Yale Tuition Postponment Plan, le Duke University Deferred Tuition Plan, etc. qui contiennent des caractéristiques très intéressantes. Au Québec, Farine proposa un fonds d'auto-financement, dont l'argument principal est qu'il est normal que ceux qui retirent le plus d'avantages de l'instruction sous forme de revenus élevés, couvrent les coûts de leur formation selon la progression de leurs revenus. ${ }^{13}$ II proposait alors de créer un fonds de subvention des universités alimenté par un impôt progressif spécial prélevé sur le revenu des bénéficiaires de la formation universitaire. Néanmoins, les contraintes politiques et financières ne sont pas à minimiser dans ce genre de projet. Le programme d'assistance financière qui a fait l'objet d'un grand intérêt est CORSAP (Contingent Repayment Student Aid Program) proposé en 1969 par G. Cook et D. Stager au Comité d'aide aux étudiants de l'Ontario. ${ }^{14}$ Il serait trop long de présenter ici le programme dans son détail. Il s'agit essentiellement d'un prêt octroyé par l'intermédiaire du gouvernement. L'originalité du programme proposé réside avant tout dans le mode de remboursement qui s'étend sur une période de trente à quarante ans ou selon une fraction fixe du revenu annuel $(.5 \%$ du revenu par $\$ 1,000$. empruntés, par exemple). Le programme favorise l'accessibilité à la formation post-secondaire en ce qu'il n'impose pas un fardeau intolérable sur les épaules des étudiants. Néanmoins, comme pour le "Fonds d'auto-financement des frais de scolarité universitaires" suggéré par Farine, la mise en marche de CORSAP implique des conditions et des contrats financiers entre les autorités fédérales et provinciales qui, du point de vue politique et financier, sont difficilement réalisables.

\section{Le service des prets et bourses aux etudiants du Quebec}

Le Québec n'est pas le seul à vouloir amender ses politiques concernant l'aide à accorder aux étudiants. L'Ontario a revisé en 1973 son régime d'aide aux étudiants, mais la discussion se poursuit, à savoir si les changements opérés ont permis plus d'accessibilité à la formation post-secondaire. ${ }^{\text {is }}$ En 1974, la Commission Royale de la Nouvelle-Ecosse a publié son rapport sur la réforme du financement universitaire, et la participation des étudiants au financement de leur formation semble être l'un des sujets les plus discutés. ${ }^{16}$

Certaines modifications ayant été apportées en automne 1974 au système de Prêts et 
Bourses du ministère de l'Education du Québec et des modifications additionnelles ayant été ajoutées dernièrement par le ministère, il nous est actuellement difficile de présenter une évaluation critique du système. Nous en restons donc au niveau des suggestions générales qui faciliteraient la solution des problèmes soulevés.

1) Il faudrait intervenir dans les calculs l'indexation de l'aide à l'étudiant.

2) Les modifications de 1974-1975 ont assoupli l'interprétation de résidence de l'étudiant, laquelle était fixée en fonction de la distance qui sépare l'institution d'enseignement du lieu de résidence des parents. Pour le besoin de budget, le Service décidait si l'étudiant "demeurait" ou non chez ses parents. Cette mesure est difficile à justifier dans le contexte socio-culturel actuel, surtout si on tient compte qu'elle s'applique à des jeunes ayant atteint leur majorité légale.

3) Les étudiants devaient obligatoirement contribuer une somme qui correspond à six semaines de disponibilité de travail pour les jeunes qui passent du secondaire au collégial et onze semaines pour ceux qui passent d'une année à l'autre au collège. Il y aurait lieu de revoir les exigences pour les étudiants qui fréquentent l'université, car il n'est pas assuré que l'expérience de travail permette de telles contributions. D'ailleurs, le nombre grandissant d'étudiants adultes à temps partiel détenant un emploi devrait être aussi pris en considération.

4) Le système de Prêts et Bourses a toujours pris pour acquis que les parents contribuent au financement des études de leurs enfants, même si le revenu familial était bas. Depuis 1974-1975, une famille dont le revenu est au-dessous de $\$ 4,500$. n'est pas considérée comme pouvant contribuer aux études de leurs enfants. Ce montant devrait être augmenté et indexé.

5) Le système actuel privilégie uniquement la formation en institution et à plein temps. Des modifications s'imposent si l'on considère la formation dans le cadre des cours à temps partiel et dans le cadre de l'éducation des adultes.

6) Il existe peu de programmes de bourses permettant d'orienter les étudiants vers les secteurs d'études prioritaires du point de vue social et économique.

7) Le remboursement des prèts s'effectue par versements fixes durant une période de temps ne pouvant excéder dix ans pour des prêts de $\$ 5,000$. et plus. La période relativement courte de remboursement et le fait que les versements soient fixes posent des difficultés aux nouveaux diplômés, alors que leur salaire est encore modeste. Une période de vingt ans et le remboursement progressif en fonction du revenu faciliteraient la solution du problème. ${ }^{17}$

8) Le Rapport Wright en Ontario et la Commission Royale de la Nouvelle-Ecosse recommandent que l'aide soit offerte principalement aux étudiants du premier cycle. ${ }^{18}$ Cette recommandation se base sur l'évidence que l'allongement des études rend l'individu plus conscient des bénéfices futurs de sa formation. ${ }^{19}$. Etant plus informés sur la situation du marché du travail, les étudiants de maîtrise ne vont probablement pas sousinvestir dans leur formation. Ce sont ceux du premier cycle universitaire qui ont besoin d'encouragement afin de poursuivre leurs études pour leur propre bénéfice et les biens qui en résultent pour la société. Cette recommandation devrait être considérée par le Québec également.

9) Il nous semble que le remboursement des prêts en proportion du revenu futur (plus de 
participation étudiante dans les coûts de leur formation) est a reconsidérer, malgré les difficultés d'application qui se posent au premier abord.

\section{Sommaire}

L'un des objectifs du gouvernement est d'assurer que les contributions privées et publiques soient faites en fonction de la rentabilité privée et sociale de la formation. Ceci nous amène à souligner que les principes d'efficacité économique et d'équité ne suggèrent pas des frais de scolarité égaux pour les divers programmes, mais plutôt des frais de scolarité directement liés aux coûts de la formation post-secondaire. Tenant compte du manque à gagner, nous sommes loin de prétendre que l'apport des étudiants n'est pas important. Cependant, la rentabilité privée de la formation demeure encore évidente au niveau individuel. ${ }^{20}$ En plus, considérant le revenu des professionnels (dans le domaine de la santé, par exemple) qui atteignent les couches économiques et administratives supérieures de la société, on peut supposer également que la majorité des étudiants considèrent leur formation comme investissement en vue de revenus importants dans l'avenir. Il est alors raisonnable d'admettre que ceux qui bénéficient de cette formation sous forme de revenus élevés, devraient supporter un pourcentage plus élevé des coûts institutionnels. ${ }^{21}$

Finalement, l'Etat doit continuer à subventionner les jeunes de familles moins aisées et à suppléer aux imperfections du marché du capital en mettant à la disposition de tous les étudiants des fonds pour leurs frais de scolarité et de subsistance. Dans le cas des imperfections du marché, le régime de prêts remboursables s'avère un des moyens à considérer.

\section{Références}

1. Bélanger, G., "Le financement de l'enseignement supérieur au Québec", L'Actualité Economique, $49 \mathrm{e}$ année, no 4 (oct.déc. 1973), pp. 467-469; Ministère de l'Education, L'éducation au Québec en 1972, p. 23; L'éducation au Québec en 1973, p. 27; L'éducation au Québec en 1974, p. 27.

2. The Learning Society, Report of the Commission on Post-Secondary Education in Ontario (The Wright Report). Toronto: Ministry of Government Services, Ontario, 1972; Peitchinis, S. G., Financing Post-Secondary Education in Canada. Calgary: Council of Ministers of Education in Canada, 1971; The Nova Scotia Royal Commission on Education, Public Services and Provincial-Municipal Relations, Vol. 1, Education. Halifax: The Queen's Printer, 1974 (Report on the Reform of University Financing).

3. Voir à cet égard, Donaldson, D. et Eaton, B.C., "Firm Specific Human Capital: A Shared Investment", The Canadian Journal of Economics, Vol. IX, no 3 (août 1976), pp. 462-472.

4. Le Conseil Supérieur de l'Education, Le collége, Rapport sur l'état et les besoins de l'enseignement collégial. Québec, juillet 1975; Université de Montréal, Statistique 1975, décembre 1975, pp. 5-10; Bélanger, P. et Rocher, G., Aspirations scolaires et orientations professionnelles des étudiants du Québec (ASOPE), 1971-1972 à 1975-1976. Université Laval et Université de Montréal.

5. Bélanger, G., déjà cité, pp. 471 et 479 ; Hettich, W., Coût, production et productivité des universités canadiennes, Conseil Economique du Canada, Etude spéciale no 14, Information Canada, 1972 , pp. 25, 76-77.

6. Une conjoncture économique défavorable amène les jeunes à poursuivre leurs études. Dans ce cas, la formation est moins coûteuse vu la probabilité accrue de ne pas trouver du travail. Voir, Handa, M.L. et Skolnik, M.L., "Empirical Analysis of the Demand for Education in Canada", in, Ostry, 
S. (Ed.), Canadian Higher Education in the Seventies, Conseil Economique du Canada, 1972, pp. 22-30; Farine, A. et Proulx, P.-P., Les diplômés de l'Université de Montréal sur le marché du travail, Dossier 5, C.R.D.E., Université de Montréal, 1974, pp. 73-74.

7. Solmon, L.C., "A Note on Equality of Educational Opportunity", American Economic Review, Vol. LX, no 4 (sept. 1970), pp. 768-771.

8. On pourra toujours consulter le travail de Proulx, P.-P., "Cost Studies in Post-Secondary Education", Stoa, Vol. 3, no 1 (1973), pp. 5-16.

9. "How Professors Spend Their Time: The 1975 Ladd-Lipset Survey of U.S. Faculty Members", The Chronicle of Higher Education (Washington, D.C.), Vol. 11 (oct. 1975), p. 2, cité dans Pédagogiques, Service pédagogique de l'Université de Montréal, Vol. 1 (janv. 1976), p. 19.

10. Bélanger, G., déjà cité, p. 467.

11. Ouellet, R. et al., "Les orientations des étudiants du cours collégial", in Ministère de l'Education, L'étudiant québécois. Défi et Dilemmes. Québec, 1972, pp. 57-93; Denis, A. et Lipkin, J., "Le Cegep promesse et réalité", Prospectives, Vol. 9, no 1 (février 1973), pp. 14-23; Hansen, W.L. et Weisbrod, B.A., Benefits, Costs and Finance of Public Higher Education. Chicago: Markham, 1969; Becker, G.S. et Chiswick, B.R., "Education and the Distribution of Earnings", American Economic Review, Vol. LVI, no 2 (mai 1966), pp. 358-369.

12. Farine, A. et Proulx, P.-P., déjà cités, pp. 47-48; Farine, A., Aspects de l'expérience universitaire et de la carriere professionnelle des étudiants. Faculté des sciences de l'Education, Université de Montréal, 1975, pp. 94-96.

13. Farine, A., "Fonds d'auto-financement des frais de scolarité universitaire", Bulletin de l'A.C.P.U., Vol. 19, no 3 (1971), pp. 13-18.

14. Cook, C.C.A. et Stager, D.A.A., "Contingent Repayment Student Assistance Program". Toronto: IQASEP, Working Paper no 7004, 1969; "Contingent Repayment Student Assistance Program: A Simulated Analy sis". Toronto: IQASEP, 1970.

15. Porter, M.R. et al., Does Money Matter? Prospects for Higher Education. Toronto: York University Institute for Behavioral Research, 1973; West, E.G., Students Loans: A reappraisal . . . with special reference to Ontario's and Canada's changing needs in educational finance. The Ontario Economic Council. Working Paper no 4/75, December 1975.

16. The Nova Scotia Royal Commission ..., Vol. 1, chaps. 61 à 65 .

17. Voir également West, déjà cité, p. 21.

18. The Learning Society, p. 147; The Nova Scotia Royal Commission . . . chap. 64, pp. 45-50.

19. West, pp. 86-87; Farine, A. et Proulx, P.-P., pp. 73-74.

20. M. F. Vaillancourt, professeur au Département des sciences économiques, Université de Montréal, est sur le point de compléter une estimation des taux de rendement de la formation au Québec.

21. Voir également, The Learning Society, p. 149. 